\title{
Zone design of specific sizes using adaptive additively weighted Voronoi diagrams
}

\author{
Pilar Moreno-Regidor ， Jésus García López de Lacalle and Miguel-Ángel \\ Manso-Callejo
}

\begin{abstract}
Territory or zone design processes entail partitioning a geographic space, organized as a set of areal units, into different regions or zones according to a specific set of criteria that are dependent on the application context. In most cases, the aim is to create zones of approximately equal sizes (zones with equal numbers of inhabitants, same average sales, etc.). However, some of the new applications that have emerged, particularly in the context of sustainable development policies, are aimed at defining zones of a predetermined, though not necessarily similar, size. In addition, the zones should be built around a given set of seeds. This type of partitioning has not been sufficiently researched; therefore, there are no known approaches for automated zone delimitation. This study proposes a new method based on a discrete version of the adaptive additively weighted Voronoi diagram that makes it possible to partition a two-dimensional space into zones of specific sizes, taking both the position and the weight of each seed into account. The method consists of repeatedly solving a traditional additively weighted Voronoi diagram, so that each seed's weight is updated at every iteration. The zones are geographically connected using a metric based on the shortest path. Tests conducted on the extensive farming system of three municipalities in Castile-La Mancha (Spain) have established that the proposed heuristic procedure is valid for solving this type of partitioning problem. Nevertheless, these tests confirmed that the given seed position determines the spatial configuration the method must solve and this may have a great impact on the resulting partition.
\end{abstract}

\section{Introduction}

Spatial partitioning entails dividing a geographic space into different units according to a specific set of criteria. The expression 'zone design' and the term 'zoning' are commonly used in the context of the geospatial sciences and land-use planning in reference to the process of partitioning a geographic space organized as a set of $N$ basic areal objects or units (postal codes, census sections, districts, etc.) that belong to a given administrative, jurisdictional or political structure. This process is carried out by clustering the units into a smaller number $M$ of zones in order to create a new spatial structure that reflects a specific set of criteria. The issue of spatial partitioning is present in a wide spectrum of applications, as 
evinced by the literature on demarcating political-electoral districts (Horn 1995, Ricca and Simeone 1997e, Mehrotra et al. 1998); delimitating socio-economic zones under a particular jurisdiction or administrative control (Openshaw and Alvanides 2001, Alvanides et al. 2002, Martin 2003); delimitating market areas or sales 'territories' (Zoltners and Sinha 1983, 2001, Ríos-Mercado and Fernández 2009); delimitating school districts (Caro et al. 2004, Ahmadi 2006); demarcating zones for delivering mobile domiciliary services, such as planning salt apportionment operations on roads (Muyldermans et al. 2002) and demarcating police districts (D'Amico et al. 2002); delimitating zones that receive energy resources (electrical power) (Bergey et al. 2003, Tiede and Strobl 2006); and finally, delimitating zones for land-use allocation and/or land acquisition and apportionment (Cova and Church 2000, Aerts and Heuvelink 2002, Williams 2002).

In all the above-cited cases, the zoning processes were determined by thematic criteria, depending on the context, and by other geographic criteria that were deemed to constitute spatial constraints. The main spatial constraints presented are as follows: (1) integrity: this criterion implies that each basic spatial unit must be assigned to a single zone, so that the units define a thorough and exclusive covering of the space to be divided; (2) contiguity: This is where each zone is built from a set of geographically adjacent units; and (3) compactness: this is the property that conditions the geometry of the zones, so that their shape approaches that of a circle or a square. Thematic criteria are usually defined with respect to one or several attributes that are used to calculate the size of the zones. These kinds of attributes, linked to basic units, are quantitative in nature and are referred to as activity measures (e.g. sales potential, number of inhabitants). Depending on these attributes, thematic criteria may establish conditions of one kind or another, for example, economic conditions, such as potential average sales and number of salespeople, and demographic conditions, such as number of inhabitants and voting population. In most cases, the main objective consists of attempting to create zones that are more or less balanced, that is, zones of similar size with respect to one or more of the given activity measures (i.e. zones with equal numbers of inhabitants, similar average sales, etc.). However, new fields of application have emerged that aim to define zones of a predetermined, though not necessarily similar, size. In addition, in the majority of these cases zones must be created based on a given set of units or seeds.

The following examples illustrate this type of zone design: (1) cereal-sheep farming and sustainable development systems: delimitating grazing polygons by clustering municipal farming plots. Each polygon is assigned to a pastoralist who keeps his flock within a certain plot. The polygons are formed around these plots, and their size or forage production capacity is proportional to each flock's feeding needs. (2) Cadastre and land-use allocation: redistributing a municipality's farming plots around value-added plots (building sites, cereal-sheep farming infrastructures, irrigation systems, etc.). A set of units is assigned to the owners around their value-added plots, with the total area being similar to the area registered in the cadastre. (3) Services: delimitating zones for business service delivery sales/maintenance by regional census tracts. The zones are created around the business headquarters in that particular region. The size or number of clients assigned to each zone is proportional to the resources available at each of the given headquarters (sales force, material resources, etc.). This type of zoning has not been sufficiently researched. The challenge is to define spatial partitions with elements of a predetermined size that are suitable for each case's given characteristics and located around a given set of locations or seeds. Within this line of research, although in a non-geographic area, Reitsma et al. (2004) and Reitsma and Trubin (2007) are worth highlighting; they proposed a method for information space partitioning in zones of a predetermined size or data volume. 
For this study we selected Castile-La Mancha's extensive cereal-sheep farming system as a case study. This kind of system produces both dryland cereals and milk/mutton all on the same units of agricultural land. Within each municipality, these small, multi-land-use plots are clustered into larger areal objects (grazing allotments or polygons) that are allocated to different pastoralists. Each plot is assigned to a single, geographically connected, grazing polygon. In order to map out the polygons, the farming plots were spatially partitioned. In this case, the size of the grazing polygons (zones) was determined by the fodder resources available (agricultural waste or other pastoral resources) so as to ensure that the flocks' feeding needs would be met and that sheep-raising activities would be sustainable.

The purpose of this research is to present a new approach that makes it possible to define zones of a predetermined size within the context of extensive farming systems; its content has been arranged as follows. Section 2 provides an overview of the models and heuristic algorithms most commonly used in solving zoning problems. Section 3 introduces the method proposed for solving this kind of problem. Section 4 presents a case study describing the criteria and constraints that must be applied. Section 5 explains and analyses the results obtained in the study area, and also discusses the methodological strengths and weaknesses. This article ends with Section 6 , which includes the conclusions of the study and a proposal for future research lines.

\section{Related work}

The issue of zone design has been widely researched since the $1960 \mathrm{~s}$, giving rise to several models or mathematical formalizations. A number of spatial and thematic conditions are established for defining zones, and these may vary considerably from one field of application to the next. The main spatial constraints are integrity, compactness and contiguity, the latter being the priority criterion in zoning problems (Shirabe 2005).

The formulation of the zone design issue is discrete, inasmuch as the zones are built as aggregates of a set of areal units or indivisible pieces. If $N$ units are to generate $M$ zones, that is, if $M<N$, there are around $M^{N}$ partitions without imposing size and contiguity constraints (Williams 2002). On the one hand, even where the $M$ value is small, the number of solutions will grow exponentially as $N$ increases; and, on the other hand, when the zones must be connected, there is no general formula to determine the total number of solutions (possible partitions). Various optimization techniques must be used in order to address these kinds of problems and find the best of all possible zoning solutions or a solution that is satisfactory and nearly optimal. In this sense, zone design may be characterized as a combinatorial optimization problem (Guo et al. 2000) that admits several models or mathematical formalizations all of which search for solutions that maximize or minimize an objective function $(F(Z))$ while complying with a number of constraints. The optimization techniques used make it possible to search for optimal or near-optimal solutions (accurate or heuristic techniques). It is possible to confirm that accurate methods have been replaced by heuristics, the latter having been used basically since 1995; at the present time, there is a trend towards metaheuristics, which combines these heuristic tools within more sophisticated frameworks (Guo et al. 2000).

In the context of optimization problems, heuristics is a function $(F)$ that helps determine which solution among a given set of possible solutions should be analysed at any given time (Weise 2009). Algorithms that use these techniques only process those elements of the search space that have been previously selected by these functions. The heuristics most commonly used to search for good or near-optimal solutions are 'hill climbing' (Horn 1995); 'simulated annealing', one of the most widely used algorithms in 
the various solution approaches (Macmillan 2001, Aerts and Heuvelink 2002, Alvanides et al. 2002, D'Amico et al. 2002, Boyland et al. 2004); 'tabu search' (Bozkaya et al. 2003); 'genetic algorithms', which are used in political districting (Bergey et al. 2003, Forman and Yue 2003, Baçâo et al. 2005); and 'greedy randomized adaptive search procedure', a less widespread algorithm than the previous heuristics (Vargas-Suárez et al. 2005, Ríos-Mercado and Fernández 2009).

Discrete combinatorial optimization zone design problems may be formalized using various mathematical models. The simplest one, though abandoned for decades, consists of envisioning the issue as a partition of sets (Mehrotra et al. 1998). The more commonly used methods are mathematical programming models (linear or mixed), graph partitioning models and cluster analysis models.

Linear programming is aimed at optimizing a linear objective function and is subject to constraints of linear equality and inequality. The following models have been developed within this line of research: integer linear programming (IP or ILP), which has been largely unsuccessful due to the difficulty of explicitly formalizing the contiguity constraint in algebraic terms; and mixed linear programming (MIP), which, from a computational point of view, is only efficient in problems involving sizes that are reduced, both in the number of basic units and in the number of zones to be generated. The most important MIP models are those created by Zoltners and Sinha (1983), applied to sales zone design; Cova and Church (2000) and Williams (2002), applied to land-use allocation or terrain acquisition; Shirabe (2005), used for generic zoning problems; and, finally, Solís et al. (2009) and Ríos-Mercado and Fernández (2009), which is applied in designing commercial zones.

The following graph partitioning models are worth highlighting: Guo et al. (2000), implemented in the multi-objective zoning and aggregation tool application; D'Amico et al. (2002), applied in demarcating police attention zones; Assunção R. et al. (2006), used to design socio-economic zones; and Tavares-Pereira et al. (2007), used in multiple criteria districting problems.

As far as cluster analysis models are concerned, several works are worth citing: Haining et al. (2000), applied in developing the spatial analysis in a geographic information system (GIS) environment system; Tiede and Strobl (2006), used in designing an algorithm to define energetically independent zones; and Ochoa et al. (2009), which is a method of implementing zoning systems in which units associated with demographic data come into play.

Many of the models and algorithms described are efficient only when applied to problems in which the number of zones $(M)$ and/or basic units $(N)$ is reduced, or that have a set of less restrictive spatial conditions, as in cases where the centres of the regions are predetermined. For example, the maximum values mentioned for $M$ and $N$ are as follows: in the MIP models, $M \leq 10$ and $N \leq 500$ (Ríos-Mercado and Fernández 2009); in the graph partitioning models, $M \in[10$. .100] and $N \leq 600$ (Guo et al. 2000); and in the cluster models, $M \in[5$. . ] and $N \leq 5000$ (Ochoa et al. 2009). These limitations, among others, have encouraged the search for other alternatives that use spatial information and computational geometry methods, such as Voronoi diagrams.

Voronoi diagrams have been used in spatial partitioning problems across several disciplines, including geospatial science. They have been applied in the fields of economic and urban geography, for example, in market zone analysis (Okabe et al. 2000), in retail trade zone design (Boots and South 1997) and in solving service location and positional optimization problems (Okabe and Suzuki 1997, Okabe et al. 2000). Given the complexity of the problems in question, generalized Voronoi diagrams (including power Voronoi diagrams, additively weighted Voronoi diagrams (AWVDs) and multiplicatively weighted 
Voronoi diagrams (MWVDs)) have been used in addition to ordinary Voronoi diagrams (OVDs). The former have been applied in urban planning projects (Boots 1975, Huff and Lutz 1979); in trade zone design (Boots and South 1997); in transportation and logistics problems (Galvão et al. 2006, Novaes et al. 2009); in delimitating zones according to socio-economic variables (Mu 2004, Ahmadi 2006, Mu and Wang 2006); and in political districting (Ricca et al. 2008).

This review's main conclusion is that the complexity of these various problems requires specific algorithms for each field of application, which makes it difficult to implement 'universal' methods. In addition, there is a need for methods that make it possible to design zones of a predetermined size depending on the context.

\section{Proposed method}

The Voronoi diagram is a commonly used method of spatial regionalization around a predetermined set of points. Given a finite set $P=\left\{p_{1}, \ldots, p_{m}\right\}$ of points on the Euclidean plane, called generators, with $m \geq 2$, the Voronoi diagram of $P$ is defined as a collection $V=\left\{V_{1}, V_{2}, \ldots, V_{m}\right\}$ of plane subsets known by the name of Voronoi regions, polygons or cells. Each $V_{i}$ is the plane region containing all the points that are closer to $p_{i}$ than to any other member of $P$. Points that are equidistant to two or more $P$ elements define the regions' borders. Formally, $V\left(p_{i}\right)$ is the Voronoi cell of point $p_{i} \in P$, such that

$$
V\left(p_{i}\right)=\left\{x \in R^{2}|\quad| x-p_{i}|\leq| x-p_{j} \mid \quad, \quad \forall 1 \leq j \leq m, i \neq j\right\}
$$

where | denotes a distance function. Where the distance is defined by Euclidean metrics (represented by \|\| ), adjacent regions with rectilinear borders are generated, and this defines an OVD.

Another type is the weighted Voronoi diagram (WVD). To build the plane tessellation, the WVD uses a function of assignment instead of a metric function (Okabe et al. $2000)$. Let $P=\left\{p_{1}, \ldots, p_{m}\right\}$ be a set of points on the Euclidean plane, and $w_{i}$ the weight of $p_{i}, \forall 1 \leq i \leq m . d w\left(x, p_{i}\right)$ denotes the weighted distance between $x$ and $p_{i}$, which in the context of the WVD depends on the $w_{i}$ value for point $p_{i}$. The definition of $d w\left(x, p_{i}\right)$ determines the kind of weighted diagram, such as the MWVD or AWVD type. For example, $d w\left(x, p_{i}\right)=\left\|x-p_{i}\right\|-w_{i}$ is an additively weighted distance, with $\left\|x-p_{i}\right\|$ being the Euclidean distance between $x$ and $p_{i}$. Any one of the WVD allows partitioning of a twodimensional space, considering both the position and the weight of each generator, seed or point of interest.

The most commonly used WVDs in zone design problems are multiplicatively weighted ones. In this case, a region $V\left(p_{i}\right)$ will be connected only if the weights of adjacent regions have values equal to or higher than its own weight $w_{i} \leq w_{j}, \forall 1 \leq j \leq m, i \neq j$. When the sizes of the regions are predetermined, compliance with this condition may be problematic or even impossible. For this reason, our approach to generating zones of a predefined size is based on AWVD, more specifically on a discrete version of the adaptive additively weighted Voronoi diagram (AAWVD), which uses a function of distance based on the metrics of the shortest path. In this case, the generators will be the centroids of the basic units corresponding to the seeds of the zones, and their weights are calculated iteratively until every zone reaches the predetermined size.

Given these characteristics, a partitioning method is proposed for a discrete twodimensional Euclidean space defined for a finite set $X=\left\{x_{1}, \ldots, x_{n}\right\}$ of points, with 
$n=$ number of centroids of the basic units in $m$ zones $\mathbf{z}_{\mathbf{i}}$, one for each generator point $\mathbf{p}_{\mathbf{i}}$, $p_{i} \subset \mathrm{X}$, characterized by its corresponding weight $\boldsymbol{w}_{\boldsymbol{i}}$. The zones shall have a predetermined or target size $\mathbf{A}\left(\mathbf{z}_{\mathbf{i}}\right)$. The proposed method establishes the following conditions:

(1) Integrity. Every point $x_{j}$ belongs to one single zone $z_{i}$.

(2) Inclusive regions, $I: p_{i} \in \mathrm{z}_{i}$. Every generator $p_{i}$ is contained in the zone $\mathrm{z}_{i}$ it generates.

(3) Contiguity. The zones $z_{i}$ are geographically connected.

The use of a metric based on the shortest path allows regions to be geographically connected. To calculate the shortest path between two points of the territory to be partitioned, a function of distance,

$$
d w\left(x, p_{i}\right)=d s p\left(x, p_{i}\right)-w_{i}
$$

is used with the constraint of having to be entirely contained in its interior. The distance between a generator $p_{i}$ and any other point $x_{j} \in X$ is the shortest connected path between those points; it is represented by $d s p\left(x_{j}, p_{i}\right)$ and defined by the sum of Euclidean distances between the centroids of adjacent units from unit $i$ to unit $j$. To calculate $d s p($, ) the Dijkstra algorithm is applied.

(4) $\nabla p_{i}=0$. A generator's position is predetermined. It is an input data and cannot change.

(5) Zones of a predefined size. For each zone $z_{i}$, a target size $A\left(\mathrm{z}_{i}\right)$ is specified. Its calculated size $a\left(\mathrm{z}_{i}\right)$ is the (total) sum of the attributes (activity measure) of basic units contained. Because of the algorithm's convergence, a maximum $\varepsilon$ per cent variation is accepted with respect to the target size, so that the maximum relative error of this variable complies with $\left|e_{i}\right| \leq 0.01 \times \varepsilon$.

An AAWVD consists basically of repeatedly solving a traditional AWVD, updating weights at each iteration based on the weights and errors in the previous iteration. This error is calculated as a function of the difference between target values $A\left(\mathrm{z}_{i}\right)$ and the values $a\left(z_{k, i}\right)$ obtained at each iteration $k: a\left(z_{k, i}\right)-A\left(z_{i}\right)$.

The weights take an initial value, $w_{0, \mathrm{i}}=0$, that is updated at each iteration $w_{k+1, i}=$ $w_{k, i}+\Delta w_{k, i}, \forall k \geq 0,1 \leq i \leq m$, where $w_{k, i}$ and $w_{k+1, i}$ are the weights of generator $p_{i}$ at iterations $k$ and $k+1$, respectively, $\Delta w_{k, i}$ is the weight increment $w_{k, i}$ at iteration $k$ and $m$ is the number of generators. $a\left(z_{k, i}\right)$ is defined as the size, expressed in terms of attribute (activity measure) units, assigned to generator $p_{i}$ at iteration $k$, and $A\left(z_{i}\right)$ is the target size, expressed in the same units, for generator $p_{i}$. The value of $\Delta w_{k, i}$ will be positive if the size $a\left(z_{k, i}\right)$ assigned to generator $p_{i}$ is smaller than the target size $A\left(z_{i}\right)$, and $\Delta w_{k, i}$ will be negative otherwise. If the relative error of generator $p_{\mathrm{o}}$ at iteration $k$ is called

$$
e_{k, o}\left(e_{k, o}=\frac{a\left(z_{k, o}\right)-A\left(z_{o}\right)}{A\left(z_{o}\right)}\right)
$$

the initial formula for calculating the weight increment is

$$
\Delta w_{k, i}=\sum_{j \neq i=1}^{m}\left(e_{k, j}-e_{k, i}\right)
$$


One of the properties of AWVD is that it can form empty zones, so that the $p_{i}$ Voronoi cell will be an empty set if there is a $p_{j}$ such that $w_{j}-w_{i}>$ $\left\|p_{j}-p_{i}\right\| \quad \forall 1 \leq j \leq m, \quad i \neq j$. In order to prevent the difference between any pair of weights from exceeding the distance between their generators, which would result in one of the zones being removed, the value of each difference $\left(e_{k, j}-e_{k, i}\right)$ is divided into the distance $d s p\left(p_{i}, p_{j}\right)$ between those generators:

$$
\Delta w_{k, i}=\sum_{\substack{j=1 \\ j \neq i}}^{m} \frac{\left(e_{k, j}-e_{k, i}\right)}{d s p\left(p_{i}, p_{j}\right)}
$$

Finally, a global parameter, $L$, is introduced to control the algorithm's speed of convergence:

$$
\Delta w_{k, i}=L \times \sum_{\substack{j=1 \\ j \neq i}}^{m} \frac{\left(e_{k, j}-e_{k, i}\right)}{d s p\left(p_{i}, p_{j}\right)}
$$

With the exposed criteria in mind, the following formula for calculating adaptive weights is obtained:

$$
w_{k+1, i}=w_{k, i}+\Delta w_{k, i}=w_{k, i}+L \times \sum_{\substack{j=1 \\ j \neq i}}^{m} \frac{\left(e_{k, j}-e_{k, i}\right)}{d s p\left(p_{i}, p_{j}\right)}
$$

To calculate the corresponding weighted distances to the centroids, $d w\left(x, p_{i}\right)$, Equation (7) is applied repeatedly to each generator until (1) the value of a parameter measuring the 'goodness' of the adjustment reaches a threshold defined by the user or (2) a maximum number $(T)$ of iterations are carried out in order to avoid an infinite loop. The 'goodness' of the adjustment is measured by an expression based on the absolute values of the relative errors in the zones: $\left|e_{k, i}\right| \leq 0.10,1 \leq i \leq m$, where $e_{k, i}$ is the relative error of generator $p_{i}$ at iteration $k$. In this case, the stop condition implies that the absolute value of the errors $e_{k, i}$, obtained at iteration $k$, is below $10 \%$ of its corresponding target value $A\left(z_{i}\right)$, that is, the process will stop if it is verified that $0.9 A\left(z_{k, i}\right) \leq a\left(z_{k, i}\right) \leq 1.1 A\left(z_{k, i}\right), \quad 1 \leq i \leq m$.

Finally, faced with the need to calibrate the $L$ parameter, included in the formula for calculating adaptive weights, a series of experiments were carried out on the case study. These experiments are described in Section 4.

\section{Case study}

The case study area is located in Castile-La Mancha (Spain). It extends over three municipalities in the province of Toledo: El Romeral (7964 ha; 9240 plots), Lillo (15,143 ha; 10,429 plots) and Corral de Almaguer (32,715 ha; 12,244 plots). The data sources used are (1) the Cadastre Office's mural cadastre, which lists plots in the three municipalities in vector format (2006); (2) the Sistema de Información Geográfica de Parcelas Agricolas (SIGPAC) Project ${ }^{1}$ consisting of a database of agricultural uses for plots in the study area (2006); (3) Virtual Cadastre Office, ${ }^{2}$ whose data were used in the editing and review processes.

The plain topography and the base-rich nature of the soils allow extensive cultivations that currently cover $95 \%$ of the study area. In addition, a traditional agro-pastoral system 
(sheep flocks) uses food resources including non-arable land (natural pastures, shrubsteppe vegetation, scrubland, etc.) and agricultural waste (the stubble from cereals and legumes, as well as fallow land). Plots used for growing olives or vineyards or for irrigation are excluded by law from use in grazing. In this region, the average size of landholdings is less than $10 \mathrm{ha}$, and property is frequently split into non-adjacent plots (Figure 1a).This is the reason why most breeders cannot maintain their own flocks. Within each municipality, Local Grazing Commissions (made up of landowners, farmers and the administration) manage pastoral resources, which are grouped by clustering the plots to form grazing allotments or polygons (Caballero 2001). The size of these polygons, assigned to landless pastoralists, is based on their capacity to produce fodder, so they are allocated to flocks whose feeding needs must be met.

In this context, the zoning problem is determined by thematic and spatial criteria. Thematic criteria determine the polygons' target size. This value is established with a tolerance range in order to promote the algorithm's convergence on a solution. Spatial criteria establish the constraints of integrity (each plot is assigned only to a single polygon) and contiguity (every polygon must be a geographically connected region made up of adjacent plots); they also determine the existence of fixed seeds (sheepfolds or places where the flocks are penned and guarded from bad weather) or generators for polygon formation. Moreover, it is necessary to consider other spatial criteria derived from the plots' specific geometry and from the use of a Voronoi diagram as part of the method.

\subsection{Thematic criteria}

In order to properly manage the study zone, it is necessary to quantify the pastoral resources linked to the plots. This quantification is called forage area equivalent (FAE), and it is

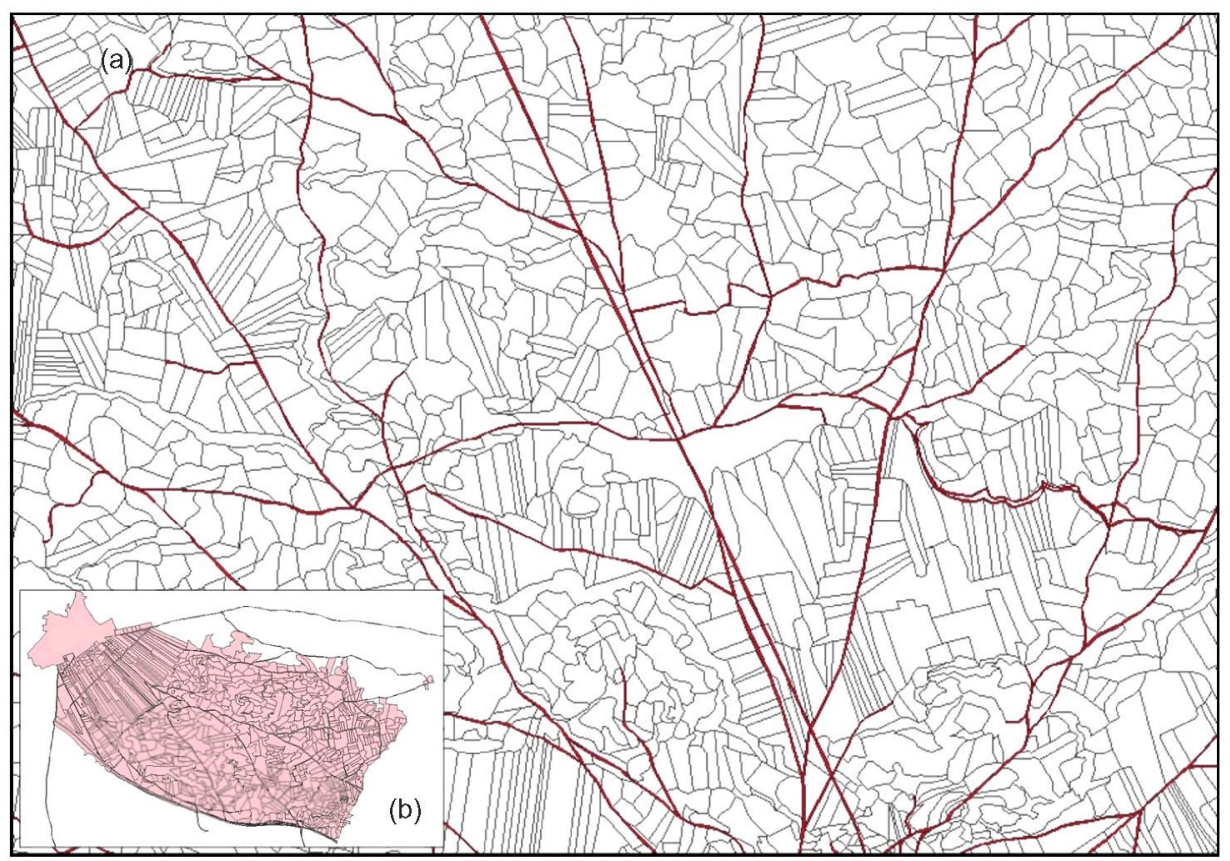

Figure 1. (a) Cadastral plots in the study area (pathways in red colour). (b) Anomalous grazing polygon, including pathway plots with their original geometries. 
calculated as a function of the type of crop and the area it occupies. A normalized forage coefficient (FC) is assigned to each crop, indicating the number of sheep that may be fed over the course of 1 year with the yield from 1 ha of that crop. The value range for this coefficient varies from 0 (unproductive land uses) to 10 (forage crops like alfalfa). Given a plot $p_{j}$ whose crop type has a forage coefficient $F C_{p j}$ and occupying an area $S f_{p j}$, its yearly forage production $F A E_{p j}$ is obtained by $F A E_{p j}=S f_{p j} \times F C_{p j}$.

Municipalities manage pasture resources on farming plots by clustering these plots into polygons with sizes that are suitable for different sized flocks. The size of the polygons is calculated based on the plots' FAE, and its error is conditioned by the error of this attribute. A plot's FAE depends on its area and on the FC of the crop planted on it. The area may be calculated with greater precision than the $\mathrm{FC}$, so the relative contribution of the first factor to any FAE error may be considered negligible. Based on the variability of nutrients in different types of crop, the maximum acceptable error for the plot's FAE is $10 \%$. This same criterion is applied to the size of the polygons, accepting with a tolerance threshold of $10 \%$ for this value.

\subsection{Geometric criteria}

Each municipality defines its own grazing polygons, so the cadastre plot structure of each municipality constitutes an independent set of basic units. The polygon's geometry will be determined by the geometry of its plots, including communication pathways. These types of units are narrow and elongated, and, when merged together with the remaining plots, they can generate zones with an 'anomalous' shape, as may be observed in Figure 1b.

Besides, the plots represent a dataset for the Voronoi diagram, so it will be necessary to replace its areal geometry by point geometry as the calculation is made. For these reasons, it is necessary to preprocess the original data sources in order to ensure that geometric representation complies with the following requirements:

(1) The plots (basic units) comprise a thorough, exclusive covering of the territory (municipality).

(2) The geometries of plots that are made up of roads or pathways must be broken up into smaller units in proportion to the length of contact with their adjacent plots. As a result of the partitioning process, the number of plots in each municipality increases by an average of $69 \%$.

(3) The plot areal geometries were replaced by the points corresponding to their centroids. They were used to define the Voronoi regions (Figure 2). After having calculated this diagram, the original areal geometry had to be reinstated in order to form and visualize the grazing polygons obtained.

After completing the processes described above, we obtained the necessary set of input data for the method proposed and implemented. Section 4.3 describes how the $L$ parameter was calibrated and selected.

\subsection{Choosing the L control parameter}

Parameter $L$ (Equations (6) and (7)) controls the speed of convergence. Two alternatives were considered: $L$ was either set at a fixed value or it was dynamically adjusted over the iterations. In order to choose the most appropriate type of parameter, several tests were carried out using data from the three municipalities and with a variable number of generators 


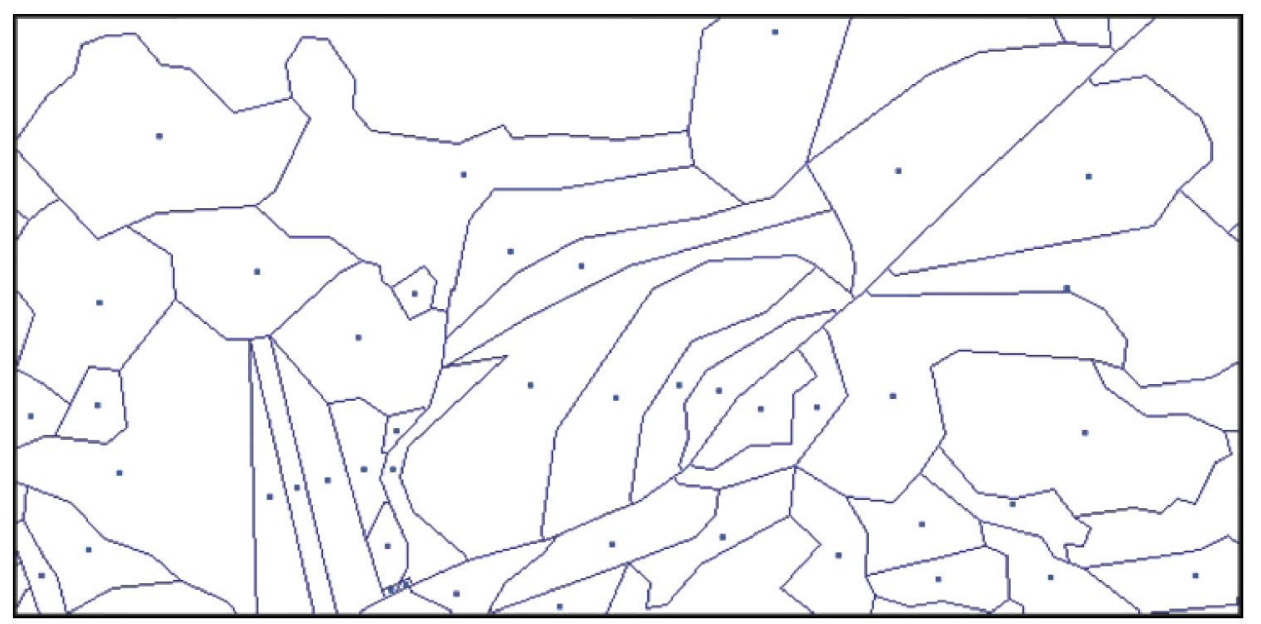

Figure 2. Geometries of plots with their centroids.

(seeds) at different locations. The aim of these tests was to evaluate whether there was a 'universal' constant applicable to all problems of this sort. The conclusions of the study carried out assigning diverse constant values to $L$ were as follows:

(1) With increasing $L$, convergence speed generally increased. For example, in partition (c) (6 zones and 17,500 units - plots) in Figure 3, the number of iterations may vary from 3151 to 23 for $L=1000$ and $L=200,000$, respectively. Yet, as observed in partitions (b) (5 zones) and (d) (9 zones), there was no convergence for $L \geq$ 5000 and $L \geq 50,000$, respectively.

In these cases, the calculation process became unstable because of the value assigned to $L$. The solution convergence process was not accelerated, but did produce a very quick initial weight adjustment, so that it began to oscillate along with the relative errors in each zone. The example in Figure 4 corresponds to a partition of 9 zones showing the errors obtained in the results, depending on the $L$-value used. When $L>50,000$, the method does not converge into a solution due to adjustments for errors in zones 1, 2 and 3 (yellow, green and blue).

(2) Impossibility of finding a 'universal' numerical constant is valid for this kind of problem. In the cases studied it was observed that the most appropriate $L$-value is part of a very broad interval $[50.300,000]$, depending on the number and geometric configurations for the generators and the number of units in the dataset.

For these reasons, using $L$ was mled out as a constant and a proposal was made to replace it with an adaptive or dynamic parameter whose value in each iteration is a function of the spatial relationship between the basic units and the degree of the zones' adjustment to their target size. To this effect, the minimum Euclidean distance $\left(D_{\min }\right)$ separating two adjacent units of the territory was calculated, as was the weighted sum of the differences between each generator's relative errors with respect to the rest. The value assigned to $L$ at each iteration was the minimum, in absolute value, of the division between these two factors. Thus, if we call $d s p\left(p_{i}, p_{j}\right)$ the distance of the shortest path between generators $p_{i}$ and $p_{j}$, and $e_{k, i}$ the relative error of generator $p_{i}$ at iteration $k$, we have the expression. 


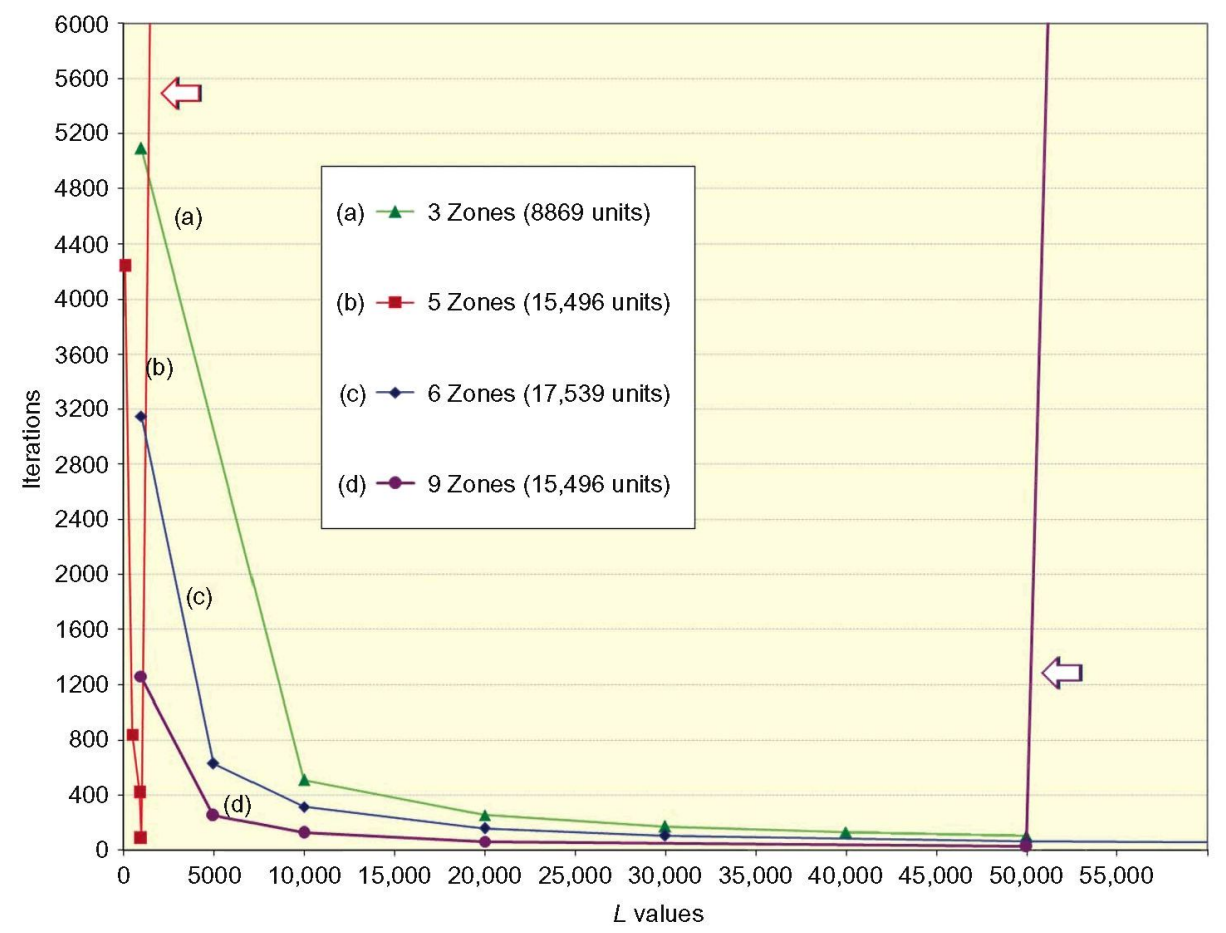

Figure 3. Variation in the number of iterations in a zoning process using different $L$-values.

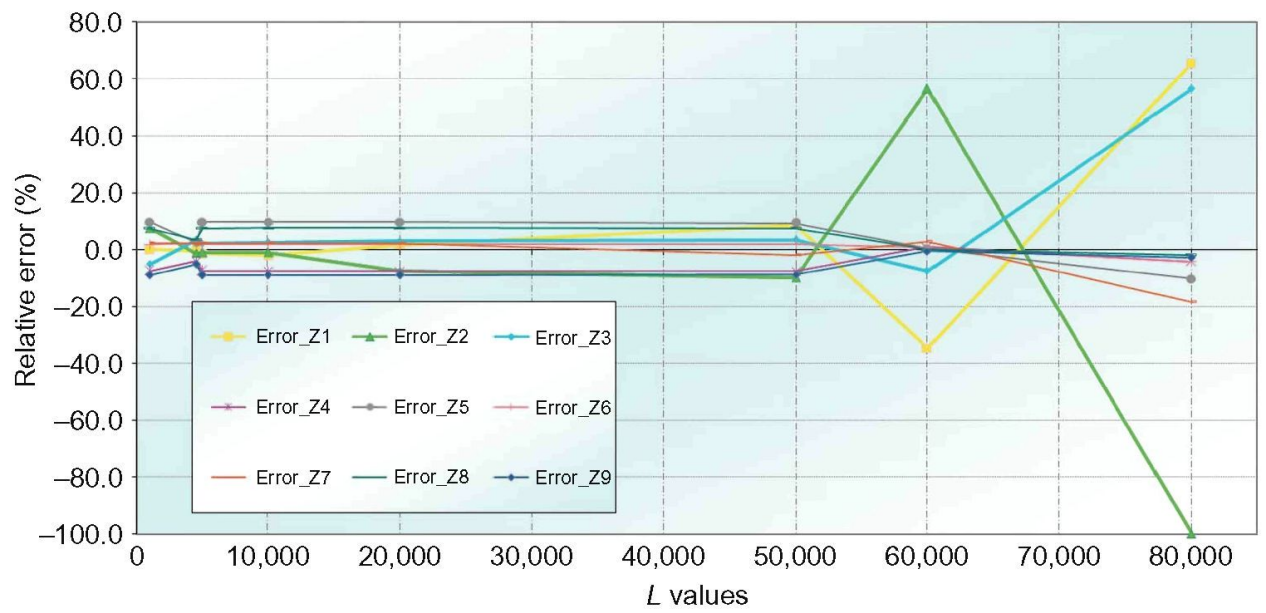

Figure 4. Variation in the relative errors obtained in a zoning process using different $L$-values (15,496 units, 9 zones).

$$
L_{k}=\operatorname{Min}\left\{\left|\frac{D_{\min }}{\sum_{\substack{j=1 \\ j \neq i}}^{m} \frac{\left(e_{k, i}-e_{k, j}\right)}{d s p\left(p_{i}, p_{j}\right)}}\right|, 1 \leq i \leq m\right\}
$$

where $L_{k}$ is the parameter value at iteration $k$ 
Replacing $L$ in Equation (7) we obtain the final equation for calculating the adaptive weights of the proposed method (AAWVD):

$w_{k+1, i}=w_{k, i}+\Delta w_{k, i}=w_{k, i}+\operatorname{Min}\left\{\left|\frac{D_{\min }}{\sum_{\substack{j=1 \\ j \neq i}}^{m} \frac{\left(e_{k, i}-e_{k, j}\right)}{d s p\left(p_{i}, p_{j}\right)}}\right|, 1 \leq i \leq m\right\} \times \sum_{\substack{j=1 \\ j \neq i}}^{m} \frac{\left(e_{k, j}-e_{k, i}\right)}{d s p\left(p_{i}, p_{j}\right)}$

\subsection{Method implementation}

An application was developed with C\# language (.NET environment) that incorporates GeoMedia Professional (Intergraph Corporation, Huntsville, United States) v.6.1's library of COM (Component Object Model) Objects in order to access the GIS environment resources and analysis functions.

The input dataset for that application is made up of all the plots or basic units in the study area stored in an Access database. For each unit there is a spatial component (areal geometry) and a thematic component (area, crop type, crop FC and degree of accessibility). The latter attribute indicates whether a unit admits flocks that got through (1) or not $(0)$. The user has to select the units, which are conceptualized as seeds or generators $\left(p_{i}\right)$ in calculating the AAWVD. For each seed, it is necessary to specify the value of the size $\left(A\left(z_{i}\right)\right)$ that should have its corresponding zone $\left(z_{i}\right)$.

The processing stages implemented are as follows:

\section{(1) Initial phase}

- Calculating the coordinates for the centroids $\left(c_{j}\right)$ and for the FAE $\left(a_{j}\right)$ of all the units or plots $\left(x_{j}\right)$.

- Generating an adjacency graph $G(V, A)$, where the vertices represent the basic units and the edges represent existing adjacencies between any given pair of units.

- Calculating distances $d s p($, ) between the vertices of the graph using the Dijkstra algorithm.

- Establishing the $T$ value (maximum number of iterations).

(2) Iterative AAWVD calculation process, using the following algorithm

(2.1) Initiate the variables:

(a) Weight of the generators: $w_{i}:=0, \forall 1 \leq i \leq m$.

(b) Number of iterations carried out: $I:=0$.

(c) Minimum Euclidean distance between any given pair of adjacent units in the territory: $D_{\min }$.

(2.2) Basic core. At each iteration $i$ :

(d) Assign $z_{i}:=\phi, \forall 1 \leq i \leq m$.

(e) For each unit $x_{j}$ represented by its centroid $c_{j}, \forall 1 \leq j \leq n$ :

- Find $p_{i}$ such that $d w\left(c_{j}, p_{i}\right) \leq d w\left(c_{j}, p_{s}\right), \forall 1 \leq s \leq m$.

- Add $c_{j}$ to $z_{i}$.

(f) For each zone or region, $z_{i}, \forall 1 \leq i \leq m$, calculate 
- Its size, $a\left(z_{i}\right)=\sum_{x_{j} \in z_{i}} a_{j}$, where $a_{j}$ is the value of the attribute (activity measure) of a unit $j, x_{j}$, belonging to $\mathbf{z}_{\mathbf{i}}$.

- Its relative error: $e_{i}=\frac{a\left(z_{i}\right)-A\left(z_{i}\right)}{A\left(z_{i}\right)}$

(g) $L$ is the control parameter.

- Calculate the variable $\operatorname{Van}_{i} \forall 1 \leq i \leq m: \operatorname{Van} x_{i}=\left|\frac{D_{\min }}{\sum_{j \neq i=1}^{m} \frac{\left(e_{i}-e_{j}\right)}{d s p\left(p_{i}, p_{j}\right)}}\right|$

- Calculate

$$
L=\operatorname{Min}\left(\operatorname{Vaux}_{i}\right)_{1 \leq i \leq m}
$$

(h) Calculate the new weight of each zone, $z_{i}, \forall 1 \leq i \leq m$ :

$$
w_{i}^{\text {current }}=w_{i}^{\text {previous }}+L \times \sum_{j \neq i=1}^{m} \frac{\left(e_{j}-e_{i}\right)}{d s p\left(p_{i}, p_{j}\right)}
$$

(i) $A \operatorname{ssign} I:=I+1$.

(2.3) If $I<T$ and $\left|e_{i}\right|>0.1$ for any zone $z_{i}, 1 \leq i \leq m$, turn back to Step 2.2.

(2.4) Generate the visual solution:

- Visualizing the units with a specific colour depending on the region they belong to, using two different hues to distinguish between units where flocks can get through and units where they cannot.

- Generating geometries of the regions obtained $\left\{z_{1}, z_{2} \ldots, z_{n}\right\}$.

\section{Results}

Figure 5 shows the results of two partitions, generated in municipalities within the study area, which consist of a different number of basic units (Corral de Almaguer: 21,037; Lillo: 17,539; E1 Romeral: 15,496). In the tests carried out, a variable number of generators (sheepfolds) were chosen in different locations.

In addition, in order to validate method outcomes, the adaptive $L$ parameter's behaviour was studied. Variations in the $L$-value were related to variations in the weights and errors of the different zones. According to expression (8), the initial value of $L$ at the first iteration is expected to be different for each problem and, as a general rule, it will increase progressively during the calculation. This behaviour responds to a tendency to adjustment and a gradual reduction of errors in the zones. As observed in Figure 6, when oscillations are produced in adjusting these errors (Figure $6 \mathrm{~b}$ ), $L$ goes through a similar pattern of variation, so that its continuous ascending behaviour stops and oscillates around the value reached up to that moment (Figure 6a).

The proposed method is novel not only because it uses AAWVD in a geographic context, but also because of its capability to handle a high number of areal geometries and to present spatial partitioning solutions with relative speed. For example, using a computer with an AMD Athlon 64 3400, $2.4 \mathrm{GHz}$ and 1 GB RAM, it takes about 19 minutes for the application to calculate a partition that includes 15,496 basic units and 6 zones, or 24 minutes in the case of a partition including 21,037 basic units and 4 zones.

In a significant number of cases the model developed with an adaptive $L$ makes it possible to find a zoning adapted to the starting criteria, defined by the target size and geometric configuration of the generators, instead of using a constant value. Moreover, when there 


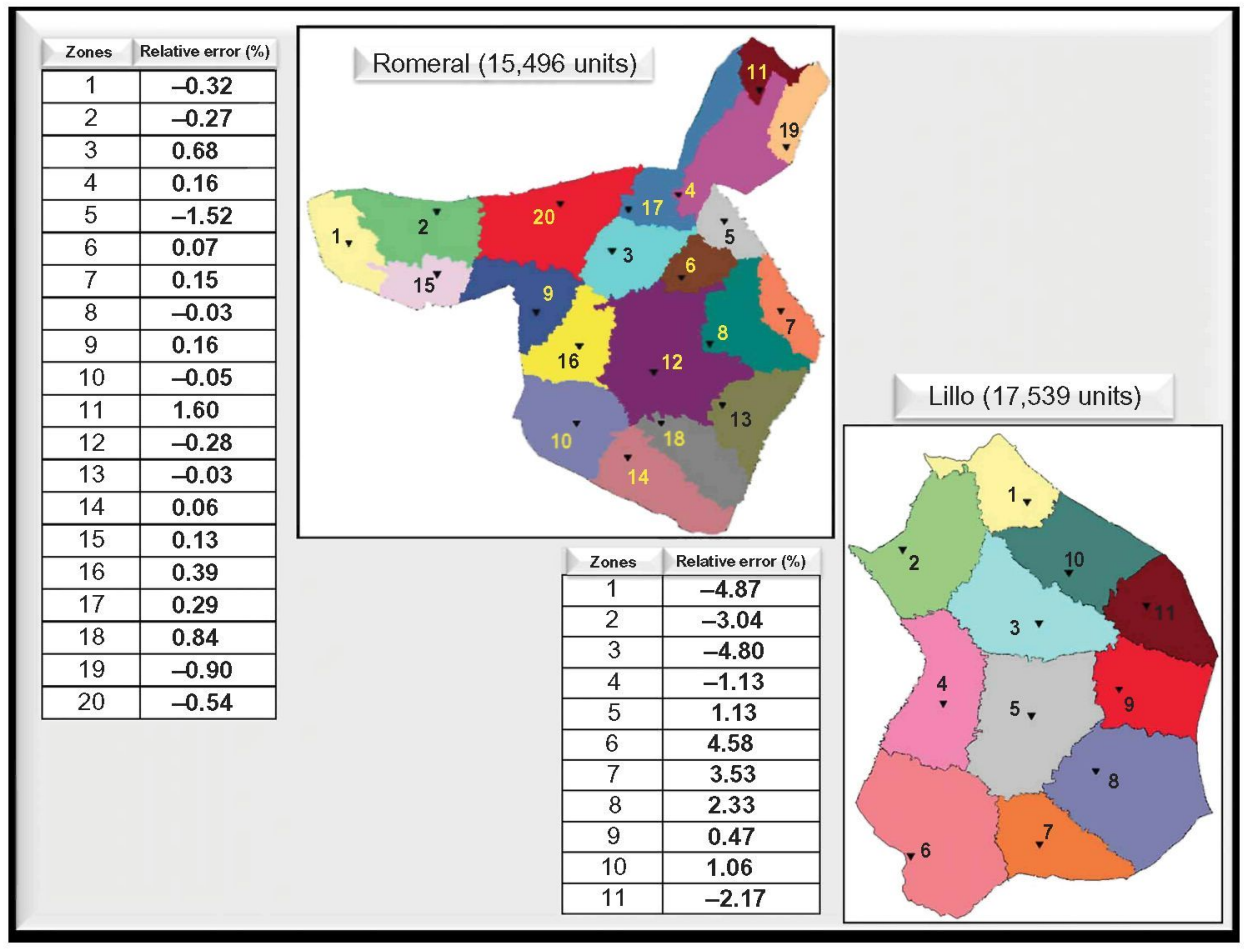

Figure 5. Examples of partitions with different numbers of units and generators.

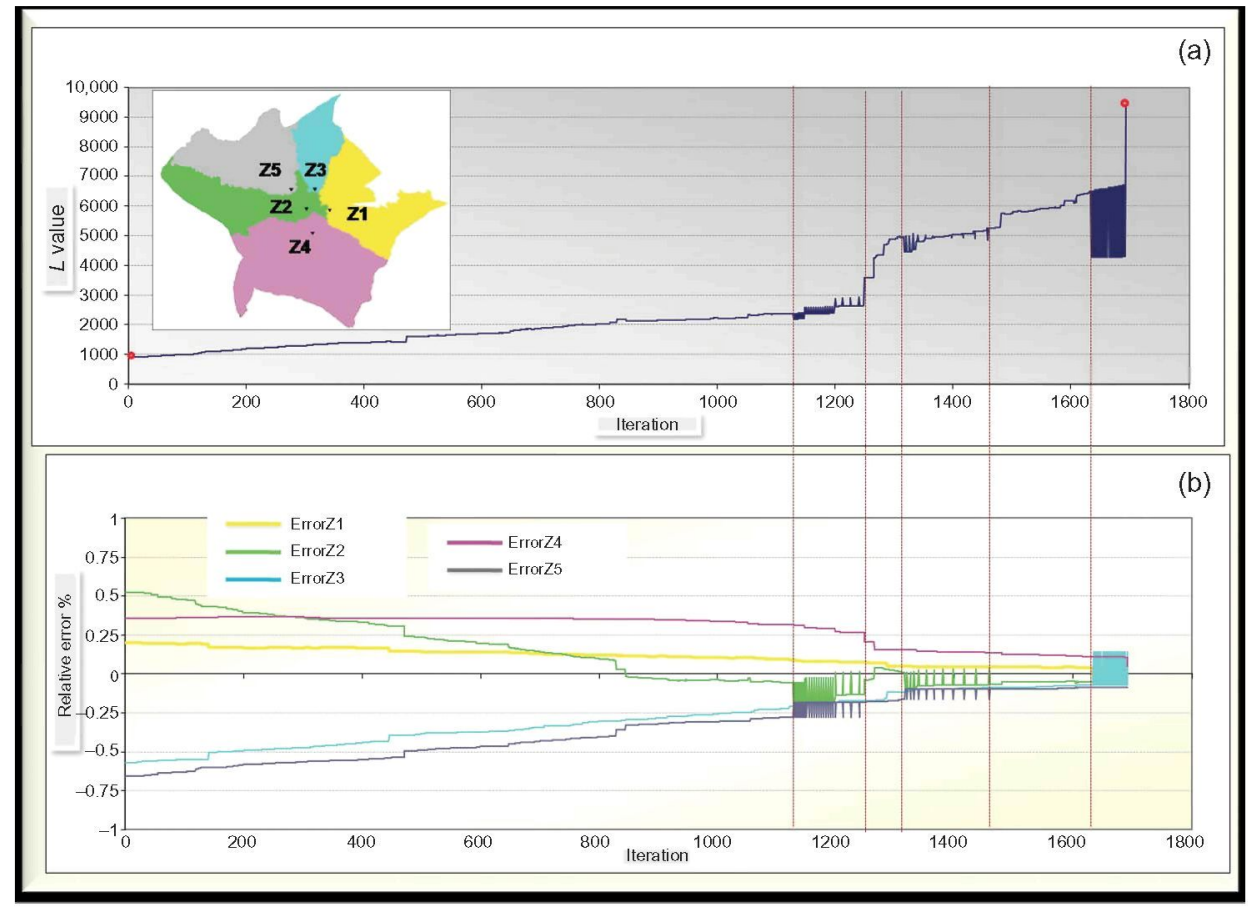

Figure 6. (a) Variation in the adaptive $L$ parameter at each iteration ( 5 zones, 21,037 units) and (b) variation in relative errors at each iteration in the zones during the calculation process. 


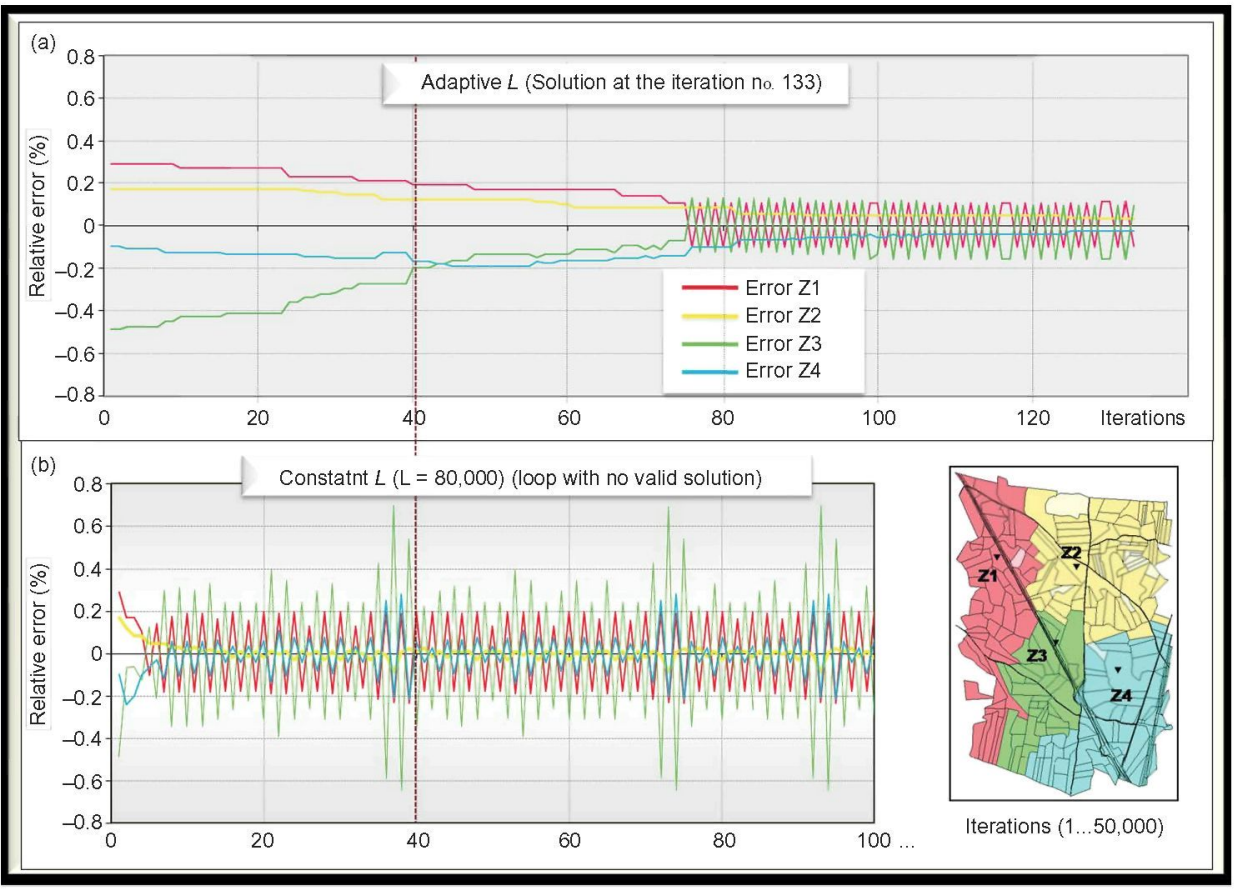

Figure 7. Variation in relative errors in the zones during the calculation process using (a) an adaptive or (b) a constant $L$ parameter.

is a solution to the problem, this adaptive parameter prevents instability in the calculation process. Figure 7 shows the variations in relative errors through consecutive iterations in the calculation process, using (Figure 7a) an adaptive $L$ or (Figure $7 \mathrm{~b}$ ) a fixed value, $L=80,000$. When $L$ is constant (Figure 7b), a very fast variation in the weights that generates a similar variation in relative errors in the zones is initially brought about, making the process unstable and therefore not generating a valid solution $\left(\mathbf{e}_{1}=\mathbf{1 9 . 5 6 \%}, e_{2}=-1.24 \%\right.$, $\mathbf{e}_{3}=-\mathbf{2 6 . 5 4} \%$ and $e_{4}=2.75 \%$; bold characters emphasizing the major errors).

Table 1. Report summary of partition errors Variation in relative errors in the zones by changing the target size or the generators' position.

\begin{tabular}{|c|c|c|c|c|c|c|c|c|c|}
\hline & \multicolumn{9}{|c|}{ Zones (territory: 15,496 units) } \\
\hline & Z1 & $\mathrm{Z} 2$ & $\mathrm{Z3}$ & Z4 & Z5 & Z6 & $\mathrm{Z7}$ & $Z 8$ & Z9 \\
\hline $\begin{array}{l}\text { (A) Relative error } \\
\mathbf{e}_{\mathbf{i}}(\mathbf{\%}) \text { (first } \\
\text { zoning) }\end{array}$ & 20.80 & -21.40 & -0.51 & -0.15 & -2.04 & -0.44 & -0.71 & -0.13 & -0.14 \\
\hline $\begin{array}{c}\text { Changes in size } \\
\text { FAE (\%) }\end{array}$ & -9.3 & -28.2 & -19.2 & 12.2 & -32.5 & -28.6 & -14.3 & 27.5 & 39.8 \\
\hline Changes in $X(\mathrm{~m})$ & - & - & - & - & -1023 & -1047 & - & - & 1206 \\
\hline Changes in $Y(\mathrm{~m})$ & & & & & -248 & 518 & - & - & 619 \\
\hline $\begin{array}{l}\text { (B) Relative error } \\
\mathbf{e}_{\mathbf{i}}(\%) \text { (second } \\
\text { zoning) }\end{array}$ & -3.16 & 15.48 & -10.23 & 0.13 & -1.45 & -1.41 & -0.27 & -0.04 & 0.49 \\
\hline
\end{tabular}


There are also cases in which the generators' relative spatial configuration hinders achieving a valid zoning with the proposed method. Some of these cases correspond to concentric distributions of the generators. Table 1 shows errors in a partition (row A) where some zones exceed $20 \%$ of the target size specified by the user. With the purpose of improving this result, another test was carried out modifying both the zones' sizes and the positions of the generators 5,6 and 9. Although the results obtained (row B) are not valid either, they are better adapted to the starting specifications. This solution has fewer errors than the previous ones: from $-20.8 \%$ and $21.4 \%$ values in zones 1 and 2 , we go on to $-15.48 \%$ and $10.23 \%$ in zones 2 and 3 , respectively.

This case, together with other cases analysed, demonstrates that a user of this application could adjust a partition, that is almost correct, by introducing changes in the regions' target sizes and/or small (virtual) displacements in the generators' locations, so that subsequent partitions would comply with the starting criteria. This methodology opens up a line of research aimed at improving the proposed method.

\section{Conclusions}

This study proposes a method based on a discrete version of the AAWVD that makes it possible to partition a two-dimensional space into zones of a predetermined size, taking into account the position and weight of each generator or seed. The shortest path metrics is the function used for distance measure, since its application ensures that the final solution will form connected regions. The method needs to simplify spatial representation of the basic units, replacing its area geometries with point geometries (centroids). Finally, to establish the zone limits, the reverse process of recovering the original areal geometries is carried out. To control the algorithm's convergence speed, an $L$ parameter is introduced. This study demonstrates that there is no universal numerical constant for $L$; hence, an adaptive formula is used. At each iteration, $L$ is calculated as a function of the spatial configuration of the centroids, the generators' locations and the relative errors in each zone.

Tests carried out in managing extensive farming system in three municipalities in Castile-La Mancha (Spain) demonstrated that the proposed method is valid in solving this kind of partition problem. The applied algorithm is capable of handling a high number (N) of basic areal units, generating solutions that converge in a reasonable CPU time and comply with the imposed constraints.

Although the complexity of this solution is greatly reduced when the generator's positions are fixed, in many cases, these positions impose a spatial configuration that the algorithm proposed is unable to solve, thus revealing one of the limitations of this method. It has been shown that the location of the generators has a considerable impact on the final solution, so that, as Kalcsics et al. (2005) observed, an 'inadequate' selection can hardly generate valid zones that comply with the established criteria. In most common zoning problems, the generators are not predetermined and have to be located in a phase prior to forming the zones. With the tests carried out, it is acknowledged that one of the major difficulties encountered by the solution approaches in this field was locating the generators, in agreement with the conclusions of Ricca et al. (2008).

This approach may be used in other fields of application, as described in the Introduction, since it is based on delimiting zones around a predetermined set of seeds, with a specifically established size and a threshold tolerance for algorithm convergence.

Having assessed the method's validity, we propose the following research lines:

- Search for methods for solving problems where the proposed algorithm does not generate a satisfactory solution. In these cases it is possible to redirect the algorithm 
through external aid. An external agent could adjust the zoning obtained by introducing changes in the regions' target sizes and/or small (virtual) displacements in the generators' locations, so that subsequent partitions would comply with the starting criteria.

- Evaluate the zones' geometry. The proposed algorithm considers the target size in the iterative adaptive process without considering other variables such as its compactness. To evaluate this parameter and other zone geometry parameters, we suggest incorporating the following elements in the method:

- Shape indices (circularity coefficient, perimeter-area index).

- Landscape structure indices (contagion, division, cohesion, aggregation and splitting index).

\section{Notes}

1. The SIGPAC Project (http://www.mapa.es/es/sig/pags/sigpac/intro.htm), created in 2005, is a GIS of agricultural plot identification, property of the present-day Ministry of Environment.

2. Information at https://ove.catastro.meh.es/

\section{References}

Aerts, J.C.J.H. and Heuvelink, G.B.M., 2002. Using simulated annealing for resource allocation. International Journal of Geographical Information Science, 16 (6), 571-587.

Ahmadi, F.A., 2006. Geographical study of school attendance areas using the multiplicatively weighted Voronoi method: a case of Rasht City [online]. Available from: http://giswin. geo.tsukuba.ac.jp/sis/thesis/Fatemeh_AHAMADI_NEJAD_MASOULEH.pdf [Accessed 4 May 2010].

Alvanides, S., Openshaw, S., and Rees, P., 2002. Designing your own geographies.P. Rees, D. Martin, and P. Williamson, eds. In the census data system. Chichester: Wiley, 47-65.

Assunção, R.M., et al., 2006. Efficient regionalisation techniques for socio-economic geographical units using minimum spanning trees. International Journal of Geographical Information Science, $20(7), 797-811$.

Baçâo, F., Lobo, V., and Painho, M., 2005. Applying genetic algorithms to zone design. Soft Computing - A Fusion of Foundations, Methodologies and Applications, 2 (5), 341-348.

Bergey, P.K., Ragsdale, C.T., and Hoskote, M., 2003. A simulated annealing genetic algorithm for the electrical power districting problem. Annals of Operations Research, 121, 33-55.

Boots, B., 1975. Patterns of urban settlements revisited. The Professional Geographer, 27, 426-431.

Boots, B. and South, R., 1997. Modeling retail trade areas using higher-order, multiplicatively weighted Voronoi diagrams. Journal of Retailing, 73 (4), 519-536.

Boyland, M., Nelson, J., and Bunnell, F.L., 2004. Creating land allocation zones for forest management: a simulated annealing approach. Canadian Journal of Forest Research, 34 (8), $1669-1682$.

Bozkaya, B., Erkut, E., and Laporte, G., 2003. A tabu search heuristic and adaptive memory procedure for political districting. European Journal of Operational Research, 144 (1), $12-26$.

Caballero, R., 2001. Typology of cereal-sheep farming systems in Castile-La Mancha (south-central Spain). Agricultural Systems, 68, 215-232.

Caro, F., et al., 2004. School redistricting: embedding GIS tools with integer programming. Journal of the Operational Research Society, 55 (8), 836-849.

Cova, T.J. and Church, R.L., 2000. Contiguity constraints for single-region site search problems. Geographical Analysis, 32 (4), 306-329.

D'Amico, S.J., et al., 2002. A simulated annealing approach to police district design. Computers \& Operations Research, 29 (6), 667-684.

Forman, S.L. and Yue, Y., 2003. Congressional districting using a TSP-based genetic algorithm, Lecture Notes in Computer Science, vol. 2724. Berlin: Springer, 2072-2083.

Galvão, L.C., et al., 2006. A multiplicatively-weighted Voronoi diagram approach to logistics district. Computers \& Operations Research, 33, 93-114. 
Guo, J., Trinidad, G., and Smith, N., 2000. MOZART: a multi-objective zoning and aggregation tool. In: Proceedings of Philippine Computing School Congress (PCSC), pp. 197-201.

Haining, R.P., Wise, S., and Ma, J., 2000. Designing and implementing software for spatial statistical analysis in a GIS environment. Journal of Geographical Systems, 2, 257-286.

Horn, M.E.T., 1995. Solution techniques for large regional partitioning problems. Geographical Analysis, 27 (3), 230-148.

Huff, D.L. and Lutz, J.M., 1979. Ireland's urban system. Economic Geography, 55, 196-212.

Kalesies, J., Nickel, S., and Schröder, M., 2005. Towards a unified territory design approach: applications, algorithms, and GIS integration. Fraunhofer-Institut für Techno- und Wirtschaftsmathematik ITWM, Kaiserslautern, Germany, Nr 71.

Macmillan, W., 2001. Redistricting in a GIS environment: an optimization algorithm using switchingpoints. Journal of Geographical Systems, 3 (2), 167-180.

Martin, D., 2003. Extending the automated zoning procedure to reconcile incompatible zoning systems. International Journal of Geographical Information Science, 17, 181-196.

Mehrotra, A., Johnson, E.L., and Nemhauser, G.L., 1998. An optimization based heuristic for political districting. Management Science, 44, 1100-1114.

$\mathrm{Mu}$, L., 2004. Polygon characterization with the multiplicatively weighted Voronoi diagram. The Professional Geographer, 56 (2), 223-239.

$\mathrm{Mu}$, L. and Wang, X., 2006. Population landscape: a geometric approach to studying spatial patterns of the US urban hierarchy. International Journal of Geographical Information Science, 20, 649-667.

Muyldermans, L., et al., 2002. Districting for salt spreading operations. European Journal of Operational Research, 139 (3), 521-532.

Novaes, A.G.N., et al., 2009. Solving continuous location-districting problems with Voronoi diagrams. Computers \& Operations Research, 36 (1), 40-59.

Ochoa, A., Bernabé, B., and Ochoa, O., 2009. Towards a parallel system for demographic zonification based on complex networks. Journal of Applied Research and Technology, 7 (2), 218-232.

Okabe, A. and Suzuki, A., 1997. Locational optimization problems solved through Voronoi diagrams. European Journal of Operational Research, 98, 445-456.

Okabe, A., Boots, B., Sugihara, K., Chiu, S.N., 2000. Spatial Tesselations. Concepts and Applications of Voronoi Diagrams. Chichester, UK: Wiley.

Openshaw, S. and Alvanides, S., 2001. Designing zoning systems for representation of socioeconomic data. In: A. Frank, J. Raper, and J. Cheylan, eds. Time and motion of socio-economic units. London: Taylor \& Francis.

Reitsma, R. and Trubin, S., 2007. Information space partitioning using adaptive Voronoi diagrams. Information Visualization, 6, 123-138.

Reitsma, R., Trubin, S., and Sethia, S., 2004. Information space regionalization using adaptive multiplicatively weighted Voronoi diagrams. In: E. Banissi, K. Börner, C. Chen, M. Dastbaz, G. Clapworthy, A. Faiola, E. Izquierdo, C. Maple, J. Roberts, C. Moore, A. Ursyn, and J. Zhang, eds. Proceedings of the 8th international conference on information visualization IV 2004. Los Alamitos, CA: IEEE Computer Society, 290-293.

Ricca, F., Scozzari, A., and Simeone, B., 2008. Drawing political districts by weighted Voronoi regions and local search. Mathematical and Computer Modelling, 48, 1468-1477.

Ricca, F. and Simeone, B., 1997. Political districting: traps, criteria, algorithms and tradeoffs. Ricerca Operativa AIRO, 27, 81-119.

Ríos-Mercado, R.Z. and Fernández, E., 2009. A reactive GRASP for a commercial territory design problem with multiple balancing requirements. Computers \& Operations Research, 36, 755-776.

Shirabe, T.A., 2005. Model of contiguity for spatial unit allocation. Geographical Analysis, 37 (1), $2-16$.

Solís, N., Ríos-Mercado, R.Z., and Álvarez, A.M., 2009. Modelando sistemas territoriales con programación entera. Ingenierias, 12 (44), 7-15.

Tavares-Pereira, F., et al., 2007. Multiple criteria districting problems. The public transportation network pricing system of the Paris region. Annals of Operations Research, 154, 69-92.

Tiede, D. and Strobl, J., 2006. Polygon-based regionalisation in a GIS environment [online]. In: E. Buhmann, S. Ervin, L. Jørgensen, and J. Strobl, eds. Trends in knowledge-based landscape modeling. Heidelberg: Wichmann, 54-59. Available from: http://www.masterla.de/conf/ pdf/conf2006/23Tiede_L.pdf. [Accessed 22 February 2010]. 
Vargas-Suárez, L., Ríos-Mercado, R.Z., and López, F., 2005. Usando GRASP para resolver un problema de definición de territorios de atención comercial. In : M.G. Arenas, F. Herrera, M. Lozano, J.J. Merelo, G. Romero, and A.M. Sánchez, eds. Proceedings of the IV Spanish conference on metaheuristics, evolutionary and bioinspired algorithms (MAEB), Granada, Spain, September 2005, 609-617.

Weise, T., 2009. Global optimization algorithms - theory and application - Version: 2009-06-26 [online]. Available from: http://www.it-weise.de/ [Accessed 11 March 2010].

Williams, J.C., 2002. A zero-one programming model for contiguous land acquisition. Geographical Analysis, 34 (4), 330-349.

Zoltners, A.A. and Sinha, P., 1983. Sales territory alignment: a review and model. Management Science, 29, 1237-1256.

Zoltners, A.A. and Sinha, P., 2001. Sales-force decision models: insights from 25 years of implementation. Interfaces, 31 (3), S8-S44. 